\title{
Determination of the fundamental colour sensations by the study of the distribution of the complementary colours in the chromatic circle. (Second note)
}

\section{M.A. Rosenstiehl}

To cite this article: M.A. Rosenstiehl (1881) Determination of the fundamental colour sensations by the study of the distribution of the complementary colours in the chromatic circle. (Second note), Philosophical Magazine Series 5, 11:68, 305-308, DOI: $10.1080 / 14786448108627020$

To link to this article: http://dx.doi.org/10.1080/14786448108627020

曲 Published online: 08 Jun 2010.

Submit your article to this journal $₫$

Llll Article views: 5

Q View related articles $\llbracket$ 
entitled "On Steady Motion in an Incompressible Viscous Fluid." Dr. Oberbeck calls attention to the tact that in my paper the same problem is proposed for solution that he investigated in vol. lxxxi. of Crelle's Journal. I obtained the number of Crelle referred to, and was struck by the similarity of the process in the latter part of my paper, which relates to the motion of a sphere in the fluid, and the method and results in Dr. Oberbeck's paper. For the method adopted, however, a reference to page 349 of my article will show that I did not claim absolute originality; I merely amplified a solution given by Mr. J. G. Butcher in vol viii. of the 'Proceedings' of the London Mathematical Society. The fact of having of necessity employed the methods of spherical harmonics, and also of having employed Borchardt's theorem, produces the only resemblance that exists in the two papers. As to Borchardt's theorem, I can merely say that $I$ have used it a dozen times since $I$ first became acquainted with it, in the winter of 1876 , while attending a course of lectures on electricity in the Johns Hopkins University. I have used the theorem in three different papers on the motion of viscous fluids.

Dr. Oberbeck states that we propose the same problem. This does not appear to be the case, as a comparison of equations (4) of my article with his equations of motion will show. Dr. Oberbeck limits his problem to the case of very small velocities, such that the products of the velocities and their differential coefficients can be neglected. Such a restriction obviously does not obtain in my paper. The problem which I have proposed, therefore, and of which $I$ have offered a possible solution, is much more general than that proposed by Dr. Oberbeck, and for which he obtains an exact solution. The vanishing of $\nabla^{2} \xi, \nabla^{2} \eta, \nabla^{2} \zeta$ has an entirely different significance in the two papers. In the form of the expressions obtained for the velocities in the case of a moving sphere, I cheerfully accord the priority to Dr. Oberbeck, though (from the different natures of the two problems) the quantities entering into these expressions do not have the same significance. I have only to say in conclusion that I have never seen Dr. Oberbeck's paper before today.

Hoping, gentlemen, that you may find it convenient to give this letter an early insertion in the Philosophical Magazine,

I remain, yours very respectfully,

Thomas Craig.

DETERMINATION OF THE FUNDAMENTAL COLOUR-SENSATIONS BY THE STUDY OF THE DISTRIBUTION OF THE COMPLEMENTARY COLOURS IN THE CHROMATIC CIRCLE. (SECOND NOTE.) BY

M. A. ROSENSTIEHL.

1. In studying the distribution of the red, yellow, green, and blue in the chromatic circle, I have found that there are in that circle three colours which, in relation to our eye, possess special 
properties ; they possess the characters of the fundamental sensations of Young. I am about to demonstrate that they are also the only ones to which these qualities belong.

It is by no means sufficient, as Helmholtz says*, that when mixed in suitable proportions they produce the sensation of white $f$. Stated in these terms the problem is indeterminate; but from their definition itself two other properties are deduced which singularly contract the limits between which the choice can be made. (1) When mixed two by two, they produce all the intermediate colours (even those which, like purple, do not exist in the spectrum); (2) they fulfil this condition without at the same time producing the sensation of white-or, more exactly speaking, producing it less than any other colours which could be chosen in their place.

These two conditions are precisely the opposite of those fulfilled by the complementary colours, which on being mixed two by two do not give rise to any intermediate colour, and, when mixed in suitable proportions, produce only the sensation of white. By their properties these two sorts of colours reciprocally limit one another; hence it follows that the study of the distribution of the complementary colours in a chromatic circle permits the determination of the points of that circle which correspond to the fundamental sensations.

2. With the aid of rotating disks I have songht, for each colour, its complementary; the following table gives the result (the notation employed being that of M. Chevreul):-

\begin{tabular}{|c|c|c|}
\hline $\begin{array}{c}\text { Colours equidistant } \\
\text { to the eye. }\end{array}$ & $\begin{array}{c}\text { Complementary } \\
\text { colours. }\end{array}$ & $\begin{array}{l}\text { Ratio of the } \\
\text { intervalsf. }\end{array}$ \\
\hline Orange-red .... & 5 th green.... & $6:$ \\
\hline Red & & $6:$ \\
\hline Orange......... & Blue-green & $6:$ \\
\hline Orange-yellow .. & 1st blue-green & \\
\hline Yellow & Blue & $6:$ \\
\hline Green-yellow .. & 3rd violet-blue... & $6:$ \\
\hline Green ........ & 4th violet....... & $6:$ \\
\hline Blue-green .... & Ora & $6: 19$ \\
\hline Blue..... & Yell & $6: 12$ \\
\hline Violet-blue & 4th yellow & $6:$ \\
\hline Violet .... & 3rd green-yellow. & $6:$ \\
\hline Violet-red & 2nd green & $6:$ \\
\hline
\end{tabular}

3. At first sight great irregularity is evident in the distribution

* Optique Physiologique, p. 384, \$20.

† I do not say "white light," because lights of the same appearance may not be identical from the physical point of view: there are white lights of which the compositions differ, but the eye cannot distinguish them one from another.

$\ddagger$ Between two consecutive colours of the first column of this table there are in reality intercalated five colours, designated by the numbers 1 . to 5; so that six equidistant colours correspond to each name. 
of the complementary colours : the intervals are very variable (from 1 to 19); and it seems impossible to deduce from them any important consequences. Helmholtz* also, who specially studied their distribution in the spectrum, could not trace any law. This irregularity will disappear when the results are discussed, if we take as our guide the preceding definitions. The chromatic circle upon which my experiments were made consists of three sections, the limits of which are formed by the ren, the yellow, and the blue. These three sections are not necessarily equidistant from one another; but the twenty-four colours of which each is formed fulfil that condition, as I have demonstrated in the first Note.

The following discussion, based on the assumption of equidistance, will not give rigorously exact results unless it be referred to a single section only. With this aim I select that which extends from the yellow to the blue, passing through the green, because in this portion of the chromatic circle the greatest number of complementary colours are assembled. The green, placed between the yellow and the blue, which by their mixture produce only the sensation of white, necessarily corresponds to a fundamental sensation. To fix its exact position (for we have the choice among eighteen colours of that name), I remark that with a second colour, at present unknown, but certainly situated between the red and the orangeyellow, and with a third colour, situated between the blue and the violet-blue, it must give the whole of the colours of the chromatic circle, at the same time producing the least possible sensation of white. It will therefore be situated at an equal distance from the complementaries of these two groups of colours.

At the first glance it might be believed that the question, being stated in such wide terms, would admit of several solutions. It is nothing of the kind. The complements of the nineteen colours extending from the red to the orange-yellow occupy in the circle only four consecutive numbers, or from the fourth green to the first blue-green. The complements of the blue to the violet-blue extend from the yellow to the fourth yellow. The colour which occupies the middle between the two systems is the third or fourth greenyellow. I cannot here give the process employed to determine the positions of the other two fundamental sensations; I confine myself to indicating the result.

The three colours are (to within $\frac{1}{72}$ ) the orange, the third greenyellow, and the third blue: they are equidistant from one another in the circle; and so are their complementaries - the first yellow, the blue-green, and the violet. The anomalies which $I$ have pointed

* Helmboltz compared the wave-lengths of the complementary colours (loc. cit. p. 365), in order to discover the law of their distribution. $\mathrm{He}$ did not, and could not, arrive at any result. The quality possessed by two colours of being complementary is of a purely physiological order ; while the wave-lengths (that is to say, the refrangibulities) are physical properties, which depend on the intimate nature of light: they would not cease to exist, even if we were deprived of an organ for their perception. 
out in the rough result of experiment thus disappear. The results obtained agree exactly with those recorded in my first Note. Maxwell*, who has studied the solar spectrum with the greatest care from the physiological point of view, has assigned slightly different positions to the colours which correspond to the fundamental sensations, as the following table shows:-

$$
\begin{aligned}
& \text { Maxweht. } \\
& \begin{array}{c}
\text { Place in the chromatic } \\
\text { circle. }
\end{array} \\
& \text { Third red ........ } \frac{2}{3} \text { from } C \text { towards } D \text {. } \\
& \text { Green........... } \frac{1}{4}, \text { E , F. } \\
& \text { Fifth blue ........ } \frac{1}{2}, F \quad \text {, G. }
\end{aligned}
$$
Place in the chromatic Place in the solar circle. spectrum.

As rotating disks permit us to experiment with greater precision, I believe these last data to be very near the trutht.

In brief, taking as a basis the equidistance of the colours which constitute each of the three sections of the chromatic circle, I arrive, by two analytical experimental methods, and without making any hypothesis, at proving that there exist three colours (the exact position of which I have determined) which possess relatively to our eye special properties. I have rigorously defined these properties, which coincide with those accorded by physiologists to the fundamental sensations. Thus the law of the mixture of colours, established $\dot{a}$ priori by Newton, developed by Young, Helmholtz, and Maxwell, is verified in its principles and specified in its consequences.-Comptes Rendus de l'Académie des Sciences, t. xcii. pp. 357360 (Feb. 14, 1881).

\section{ON TONES PRODUCED BY THE INTERMITTENT IRRADIATION OF A GAS. BY W. C. RöNTGEN.}

In my lectures on experimental physics I have for some time employed the following apparatus to render visible, in a simple way, the different capabilities of absorbing heat-rays possessed by the gases.

$A$ glass tube about 4 centims. in diameter and 40 centims. in length, placed horizontally, is closed at both ends with plates of

* Proceedings of the Royal Society of London, vol. x. p. 404 (1860).

+ Maxwell, moreover, operated upon only sixteen colours of the spectrum, which he mixed three by three. 